\title{
Application of an ELISA test using Schistosoma bovis adult worm antigens in travellers and immigrants from a schistosomiasis endemic area and its correlation with clinical findings
}

\author{
JAVIER PARDO ${ }^{1,2}$, JOSE LUIS PÉREZ ARELLANO ${ }^{3}$, ROGELIO LÓPEZ-VÉLEZ ${ }^{4}$, \\ CRISTINA CARRANZA ${ }^{3}$, MIGUEL CORDERO ${ }^{2} \&$ ANTONIO MURO $^{1}$
}

${ }^{1}$ From the Laboratorio de Inmunologia Parasitarna y Molecular, CISET, Facultad de Farmacia, Universidad de Salamanca, Salamanca, ${ }^{2}$ Unidad de Enfermedades Infecciosas-Medicina Interna III, CISET, Hospital Universitario de Salamanca, Salamanca, ${ }^{3}$ Dpto Ciencias Médicas y Quirúrgicas, Facultad de Ciencias de la Salud, Universidad de Las Palmas de Gran Canaria, C/Doctor Pasteur, Las Palmas de Gran Canaria, and ${ }^{4}$ Unidad de Medicina Tropical y Parasitología Clínica, Servicio de Enfermedades Infecciosas, Hospital Ramón y Cajal, Madrid, Spain

\begin{abstract}
We have recently evaluated an ELISA for the diagnosis of human schistosomiasis using S. bovis adult worm antigens (AWA $\mathrm{Sb}$ ), showing a sensitivity of $94 \%$ and a specificity of $97 \%$ for patients diagnosed by egg detection. Nevertheless, the comparison of this AWA Sb ELISA with direct parasitological findings as the gold standard could introduce a selection bias, due to the well-known lack of sensitivity of direct methods in the detection of acute schistosomiasis and of low burden infections. The objective of the present work is to compare it with parasitological methods and commercial indirect haemagglutination test using S. mansoni antigens (WA Sm IHA) in 254 immigrants and travellers with different clinical settings; in addition, to find specific bands in the EITB of different phases of schistosomiasis. The AWA Sb ELISA showed $72 \%$ of seropositivity in patients with Katayama fever, while patients with eosinophilia and genito-urinary complaints showed $27 \%$ and $93 \%$, respectively. The diagnosis yield was globally higher than direct egg detection or WA Sm IHA test with regard to the clinical setting. Finally, the utilization of EITB with S. bovis AWA permits the confirmation of diagnosis in chronic and acute phases of the disease.
\end{abstract}

\section{Introduction}

Human schistosomiasis affects 200 million people and 650 million more are at risk, most of them in sub-Saharan Africa [1,2]. Although, until recently, schistosomiasis was considered an exotic disease in Europe, a substantial increase of imported cases has been reported in the last decade. The main species involved in these cases are Schistosoma (S) mansoni, S. haematobium and S. intercalatum [3].

The diagnosis of clinical schistosomiasis is usually confirmed by parasite eggs detection in stool or urine. Nevertheless, most schistosome-infected patients are asymptomatic. In addition, parasitological methods have a low diagnostic yield in patients with acute or low-intensity infections [4]. Thus, several authors have recommended the use of additional diagnostic approaches in imported schistosomiasis [3,5-7]. Serological methods are, to date, the most effective for the diagnosis of imported schistosomiasis $[8,9]$. The use of adult worm microsomal antigens from $S$. mansoni or $S$. haematobium gives a high sensitivity through the detection of antibodies against these species. Nonetheless, the sensitivity for cases due to other schistosoma species is low $[10,11]$. Confirmatory electroimmunotransfer blotting (EITB) analysis has sometimes to be further used to allow species definition by using speciesspecific antigens [10].

We have recently evaluated an ELISA test for the diagnosis of human schistosomiasis using $\mathrm{S}$. bovis adult worm antigens (AWA Sb ELISA), which gave a sensitivity of $94 \%$ and a specificity of $97 \%$ for

Correspondence: A. Muro, Laboratorio de Inmunología Parasitaria y Molecular, CISET, Facultad de Farmacia, Universidad de Salamanca, Avda. Campo Charro s/n 37007 Salamanca, Spain. Tel: +34 923294535. Fax: +34 923294515. E-mail: ama @usal.es 
samples from patients diagnosed by parasite eggs detection. Moreover, this ELISA showed the same sensitivity for the diagnosis of S. mansoni, S. haematobium or $S$. intercalatum infections. We also showed that EITB with S. bovis adult worm antigens further allows diagnostic confirmation when the AWA Sb ELISA is positive [12]. Nevertheless, comparing the accuracy of this AWA Sb ELISA with parasitological microscopy findings gold standard - could introduce a selection bias due to the lack of sensitivity of egg detection in the acute and prepatent periods of the disease and in low burden infections [4].

The aim of the present work is to compare the diagnostic performance of the AWA Sb ELISA with classical parasitological methods and with a commercial indirect haemagglutination test in travellers and immigrants from endemic areas with different clinical settings. Furthermore, we study the utility of an EITB using AWA Sb antigens for definition of specific banding patterns of both acute and chronic schistosomiasis.

\section{Materials and methods}

\section{Patients}

75 sera from travellers with freshwater exposure (specifically bathing) and 179 sera from immigrants arriving from schistosomiasis endemic areas and attending 2 tropical medicine units (Hospital Insular de Las Palmas de Gran Canaria, and Hospital Ramón y Cajal, Madrid, Spain) were selected between 2000 and 2004 for the detection of imported schistosomiasis. A defined set of demographic, clinical and laboratory data was collected for each patient.

In order to compare the value of 3 different diagnostic tests with defined clinical findings, we divided patients into 6 different clinical groups: 1) Acute schistosomiasis (Katayama fever) - this included travellers who reported at least 3 of the 4 following clinical criteria: i) swimmer's itch; ii) temperature above $38^{\circ} \mathrm{C}$; iii) eosinophilia over $450 / \mu \mathrm{l}$; iv) any of the following symptoms or signs general syndrome, rash, digestive manifestations (nausea, vomiting, diarrhoea, liver or spleen enlargement), joint or muscle aches, headache, respiratory symptoms (cough, dyspnoea, wheezing). 2) Genitourinary tract involvement patients with urinary (dysuria, haematuria, other) or genital (haematospermia, dispareunia, other) symptoms; 3) Eosinophilia, patients with eosinophil blood counts over $450 / \mu 1$ and without clinical data suggesting any parasitic disease; 4) Asymptomatic microhaematuria, patients with a count over $5 \mathrm{RBC} / \mathrm{hpf}$ (red blood cells/field) in the urine sediment or a positive dipstick determination in asymptomatic patients; 5) Other, patients with any other kind of semiology not included in the above-mentioned groups; 6) Asymptomatic patients without clinical or biological data suggesting an imported disease.

In addition, we used sera from patients with schistosomiasis defined by eggs detection as positive control group $(n=10)$ and healthy Spanish blood donors without travel to an endemic area of schistosomiasis as negative control group $(n=30)$.

\section{Diagnostic techniques}

Parasitological methods. Examination of the presence of Schistosoma eggs in faeces was carried out in triplicate using the Kato Katz thick smear technique [13]. Schistosoma eggs excreted in urine were detected by filtration (Nucleopore polycarobonate membranes, Corning Separations, Acton MA, USA) [14].

Serological methods. Schistosoma bovis adult worm antigen (AWA Sb) preparation was performed according to López Abán et al. [15]. AWA Sb ELISA and AWA Sb EITB were carried out as described in Pardo et al. [12]. Indirect haemagglutination with soluble worm adults of Schistosoma mansoni (WA Sm IHA) was performed in 101 sera of different clinical groups, using a commercially available technique (Fumouze Laboratories, Levallois-Perret, France). Sera were considered positive when the titre was $\geq 1 / 80$. All parasitological and serological tests were double blind conducted.

\section{Statistical analysis}

Statistical tests were carried out using the SPSS 11.5 Statistical Package. Results were considered significant different when $p<0.05$. The results are expressed as means and standard deviations and percentages. $\chi^{2}$ and Fisher's tests were used to define the association between clinical variables and the parasitological data. Student's $t$-analysis of variance test and SDS post hoc were used to compare AWA $\mathrm{Sb}$ ELISA optical densities from the different clinical groups. Kruskal-Wallis and Bonferroni tests were used if the variances were not homogeneous. AWA Sb ELISA and SEA Sm IHA results were compared using $\chi^{2}$ test.

\section{Results}

Demographic, epidemiological and clinical characteristics

We selected 75 travellers and 179 immigrants, $70 \%$ of them male and $30 \%$ female. Mean age was 
$33.2 \pm 7.2 \mathrm{y}$ in travellers and $27.8 \pm 9.2 \mathrm{y}$ in immigrants. They mainly arrived from West Africa (85\%).

74 of the 254 patients (29\%) (20 travellers, $27 \%$ of their group and 54 immigrants, $30 \%$ of their group) were diagnosed with schistosomiasis using parasitological and/or serological methods (AWA Sb ELISA). The mean age of patients with schistosomiasis was $29.0 \pm 9.0 \mathrm{y}, 75 \%$ of them being male. The patients with schistosomiasis arrived mainly from occidental Africa (92\%), mainly Mali (25 cases, 32\%), and Ghana and Equatorial Guinea, with 12 cases each (15\%).

Clinical findings were: Katayama fever in 11 cases (all travellers), genitourinary tract involvement in 13 (6 travellers and 7 immigrants), eosinophilia in 95 (13 travellers and 82 immigrants), microhaematuria in 29 ( 2 travellers and 27 immigrants), and other clinical findings in 27 (2 travellers and 25 immigrants). The remaining 79 patients were asymptomatic (41 travellers and 38 immigrants).

\section{Parasitological findings}

The diagnosis of schistosomiasis was made in 32 patients (13\%) by the detection of eggs: 21 patients had S. haematobium eggs in urine, 8 patients had S. mansoni and 2 patients $S$. intercalatum eggs in faecal samples. One patient had both $S$. mansoni and $S$. haematobium eggs in faeces and urine, respectively. The diagnosis of schistosomiasis by egg detection was more frequent in immigrants than in travellers: 27 cases $(15 \%)$ vs 5 cases $(7 \%)(p<0.05)$.
The relationships between clinical/biological data and parasitological findings are shown in Table I. A statistically significant relationship between the presence of eggs in urine and both genito-urinary complaints and asymptomatic haematuria was found $(p<0.05)$.

\section{AWA Sb ELISA}

General data. The optical density (OD) in AWA Sb ELISA from patients' sera divided into the abovementioned clinical groups are presented in Figure 1. 70 patients $(27 \%)$ (20 travellers (27\% of their group) and 50 immigrants (27\% of their group)) were positive. Of the 32 patients diagnosed by egg detection, 28 were seropositive, giving a sensitivity of $87 \%$ for the AWA Sb ELISA.

Correlation with clinical findings. The OD mean for the group of patients with genital and urinary complaints $(0.99 \pm 0.35)$ and Katayama fever $(0.78 \pm 0.33)$ was the highest, with significant differences with the rest of the clinical groups $(p<0.05)$. From 11 patients with Katayama fever, 8 (73\%) were AWA Sb ELISA positive. 12 of 13 patients $(92 \%)$ with genito-urinary complaints were positive. In the group of 95 patients with eosinophilia, 26 $(27 \%)$ were AWA Sb ELISA positive. From 29 patients with asymptomatic microhaematuria, 16 $(55 \%)$ were positive. Only 3 (11\%) from the patients with other clinical manifestations had seropositivity. Finally, 5 of 79 asymptomatic patients $(6 \%)$ were seropositive.

Table I. Comparison of the diagnostic yield of the AWA Sb ELISA with classical parasitological methods in different clinical settings.

\begin{tabular}{|c|c|c|c|}
\hline \multirow[b]{2}{*}{ Clinical findings $(n)$} & \multicolumn{3}{|c|}{ Diagnostic methods $n$ positive $/ n$ available (\% positivity) } \\
\hline & Eggs (stool) & Eggs (urine) & ELISA AWA Sb \\
\hline Katayama fever $^{1}(11)$ & $0 / 11(0)$ & $0 / 9(0)$ & $8 / 11(72)$ \\
\hline Genital and urinary complaints ${ }^{2}(13)$ & $0 / 13(0)$ & $8 / 13(61)^{\mathrm{a}}$ & $12 / 13(92)$ \\
\hline Eosinophilia $^{3}(95)$ & $5 / 84(6)$ & $4 / 38(10)$ & $26 / 95(27)$ \\
\hline Microhaematuria $^{4}(29)$ & $2 / 29(7)$ & $9 / 22(40)^{\mathrm{a}}$ & $16 / 29(55)$ \\
\hline Others $^{5}(27)$ & $1 / 17(6)$ & $0 / 7(0)$ & $3 / 27(11)$ \\
\hline Non-specific/asymptomatic ${ }^{6}(79)$ & $3 / 65(5)$ & $1 / 28(3)$ & $5 / 79(6)$ \\
\hline All cases $(254)$ & $11 / 219(5)$ & $22 / 117(19)$ & $70 / 254(27)$ \\
\hline
\end{tabular}

\footnotetext{
${ }^{1}$ Katayama fever was defined in travellers with fresh water exposure and at least 3 of the 4 following clinical criteria: 1) swimmer's itch; 2) temperature above $38^{\circ} \mathrm{C}$; 3) one of the following: general syndrome, skin manifestations ... and 4) eosinophilia.

${ }^{2}$ Genito-urinary tract involvement patients with urinary (dysuria, haematuria, other) or genital (haematospermia, dispareunia, other) symptoms.

${ }^{3}$ Eosinophilia without evident parasite cause was defined if the patient presented an absolute eosinophil count $>450$ eosinophils/ $\mu$ l and did not refer to any other clinical data of a parasite infection.

${ }^{4}$ Asymptomatic microhaematuria was defined if the patient presented a count $>5 \mathrm{RBC} / \mathrm{hpf}$ in the urine sediment and did not refer to any other clinical data.

${ }^{5}$ Other, patients with any other kind of semiology not included in the above-mentioned groups.

${ }^{6}$ Non-specific or asymptomatic: absence of clinical data or analysis suggestive of schistosomiasis.

a Association statistically significant between the detection of eggs in urine and genital and urinary complaints and patients with asymptomatic haematuria.
} 
OD (492 nm)

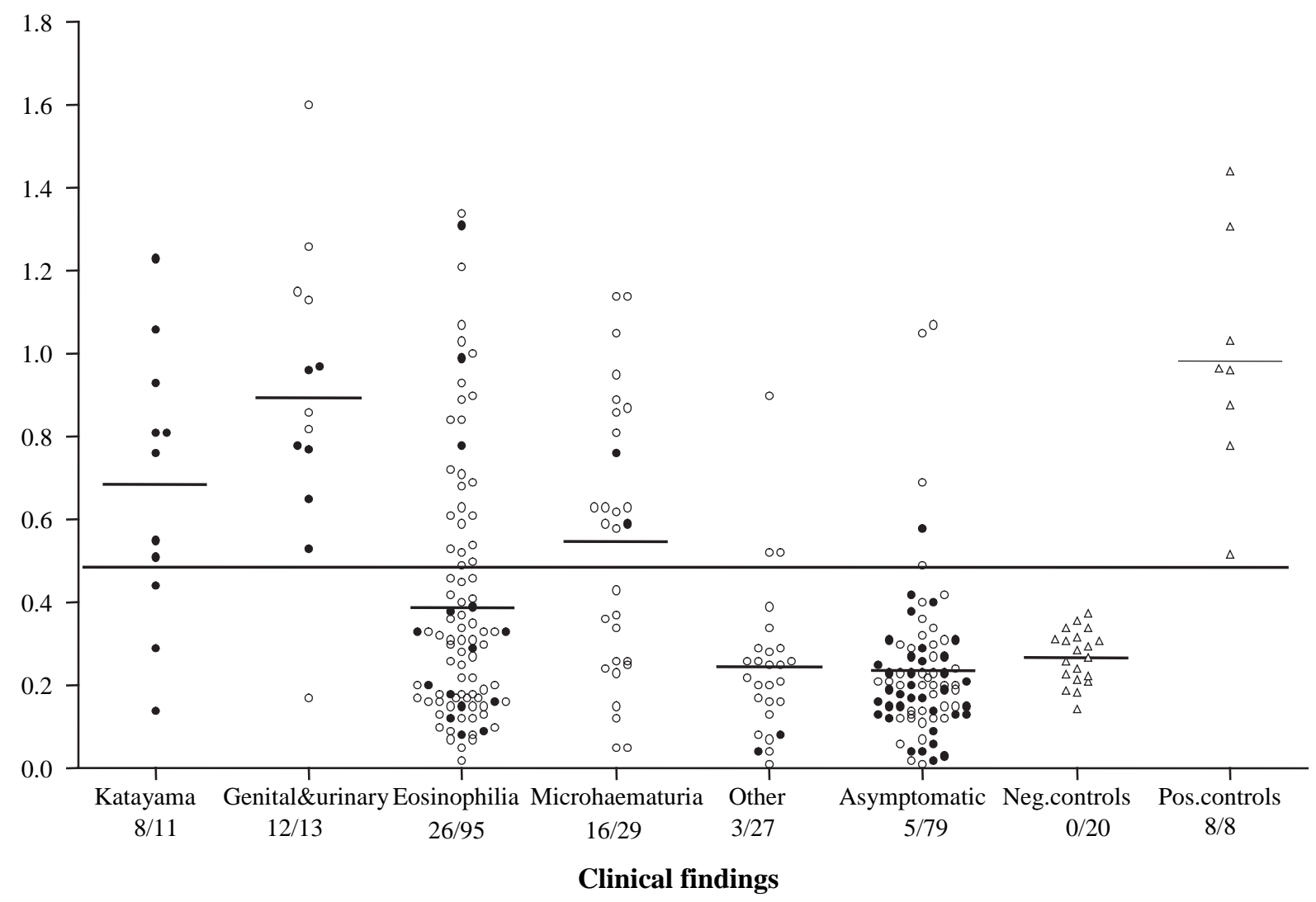

Figure 1. Optical density of sera from patients included in the study in relation with most frequent clinical findings. Number of patients seropositives/number of patients each group. Cut-off was established previously in optical density of 0.500 . Black circle = travellers, white circle $=$ immigrants. Triangle $=$ control positive and negative sera.

Comparison with parasitological findings. The AWA Sb ELISA test showed a higher number of positive samples than the parasitological test regardless of clinical findings. The OD mean of sera from patients diagnosed by egg detection and that in ELISA positive and parasitologically negative was similar $(0.85 \pm 0.31$ and $0.77 \pm 0.22$, respectively. $(p>$ $0.05)$.

Comparison with WA Sm IHA. 101 sera were also tested with a commercial assay (WA Sm IHA). In comparison, the AWA Sb ELISA was positive for a higher number of patients than the WA Sm IHA $(27 \%$ vs $18 \%, p<0.05)$. Specifically, in the Katayama fever, genito-urinary complaints and eosinophilia groups, the AWA Sb ELISA detected more positive samples than the WA Sm IHA (Figure 2). In addition, the sensitivity of the AWA Sb ELISA proved to be higher than the WA Sm IHA sensitivity in parasitologically positive patients $-8 / 8(100 \%)$ vs $6 / 8(75 \%)$ (data not shown).

\section{AWA Sb EITB}

The EITB recognition pattern from AWA Sb ELISA positive sera of patients with Katayama fever was similar to that exhibited by sera from chronic schistosomiasis positive patients (Figure 3). Of the 5 main protein clusters characteristic of schistosomiasis $(85,65,37,29,20 \mathrm{kDa}$ bands), the $37 \mathrm{kDa}$ band was recognized only by 1 serum (12\%) from the acute schistosomiasis patients' group. Sera from the remaining clinical groups with AWA Sb ELISA positive results presented a similar pattern of bands to chronic schistosomiasis patients.

\section{Discussion}

In this work we compare, in immigrants and travellers from endemic areas with different clinical settings, the diagnostic performance of the AWA Sb ELISA with a commercial indirect haemmagglutination test and parasitological methods.

We showed the utility of AWA Sb ELISA for detection of parasitological negatives cases. Thus, 


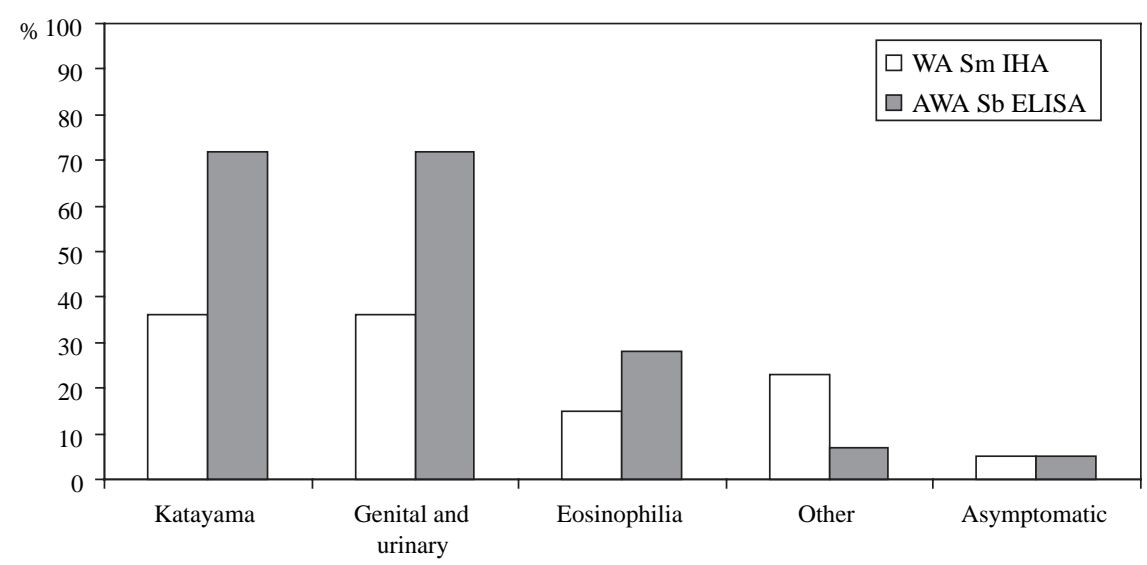

Figure 2. Diagnostic yield of indirect haemagglutination (IHA) with Schistosoma mansoni egg antigens (WA Sm IHA) and ELISA with adult whole worm of Schistosoma bovis (AWA Sb ELISA) in 101 sera of patients with different clinical findings. Sera from patients with clinical diagnosis of Katayama (11), patients with genital and urinary complaints and microhaematuria (11), patients with eosinophilia without other clinical findings (32), patients with other clinical findings (13) and asymptomatic without other clinical or laboratory findings (34).

using this test, we can detect $72 \%$ of cases with clinical suspicion of Katayama fever, while all of these cases were negatives parasitologically. The positivity of AWA Sb ELISA is higher than detected by other authors with S. mansoni soluble egg antigens in patients with Katayama fever $(25 \%-$ $50 \%$ positivity) [16,17]. Moreover, we detected additional parasitologically negative cases in the group of genital and urinary complaints, a classical syndrome of chronic schistosomiasis. Similarly, AlSherbiny et al. [10] showed that the detection of antibodies significantly improved the sensitivity of detecting schistosome eggs in patients with urinary abnormalities in an endemic area.

When a new immunodiagnostic method is evaluated, it is important to compare its performance

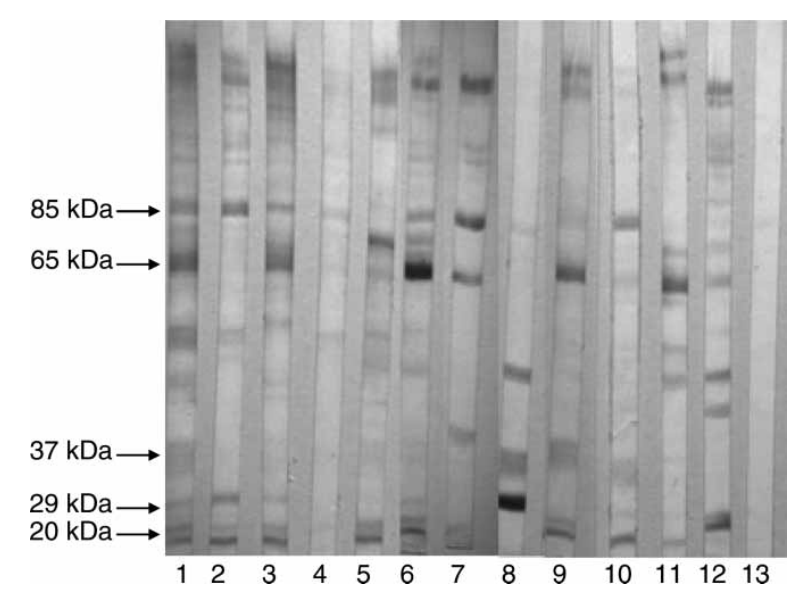

Figure 3. EITB of sera from patients who were positive by ELISA AWA Sb. Lane 1: Schistosomiasis defined by egg detection. Lanes 2-7: Patients with clinical diagnosis of Katayama fever. Lane 8: Patient with eosinophilia. Lanes 9-11: Patients with microhaematuria. Lane 12: Patients asymptomatic. Lane 13: Healthy control. with other well-known tests. In a previous work we found a high correlation between the results of the AWA Sb ELISA and an ELISA using whole S. mansoni egg antigens [15]. In this work we directly compare the diagnostic performance of the AWA Sb ELISA with a commercial indirect haemagglutination test (WA Sm IHA). This method has been shown to present the highest diagnosis yield for imported schistosomiasis [16]. As shown, we have found a higher sensitivity using the AWA Sb ELISA than the WA Sm IHA for cases defined by eggs detection. Moreover, we have detected with ELISA AWA $\mathrm{Sb}$ a higher number of cases of schistosomiasis parasitologically negative than WA Sm IHA. Thus, in the group of Katayama fever (acute schistosomiasis) and the group of genital and urinary complaints (chronic schistosomiasis), the ELISA AWA Sb detected more cases than WA Sm IHA. This higher yield could be explained by the property of the AWA $\mathrm{Sb}$ ELISA to detect antibodies against both $\mathrm{S}$. mansoni and S. haematobium [12], while serological tests performed with $\mathrm{S}$. mansoni purified antigens generally show a lower sensitivity for the diagnosis of S. haematobium infections $[10,11]$. In this regard, the interspecies variability is known to affect the diagnostic characteristics of many serological tests. The diagnostic relevance of this interspecies variability should not be overlooked, owing to the increasing number of imported S. haematobium schistosomiasis cases [3].

Finally, in our previous work we showed, using sera of patients with schistosomiasis defined by eggs, the utility of EITB with AWA Sb for confirmation of a result AWA Sb ELISA positive. In this work we detected in patients with different clinical settings mostly without eggs - the same pattern of bands as that previously defined in patients with chronic 
schistosomiasis. This fact supports the use of AWA $\mathrm{Sb}$ EITB for the confirmation of diagnosis in acute schistosomiasis.

In sumary, the application of an ELISA test using AWA $S b$ in patients from endemic areas has a higher diagnostic value for parasitologically negative cases, supporting the usefulness for the diagnosis of acute schistosomiasis. The sensitivity of AWA Sb ELISA is higher than WA Sm IHA. Finally, the utilization of EITB with S. bovis AWA allows the confirmation of diagnosis in the acute phase of the disease.

\section{Acknowledgements}

We would like to thank Mar Siles-Lucas for his review of the manuscript and G.H. Jenkins for help with the English version of the manuscript. This study was supported by Fondo de Investigaciones Sanitarias (FIS) 01/685 and Red de Investigación Cooperativa en Enfermedades Tropicales (RICET) ref: $\mathrm{C} 03 / 04$.

\section{References}

[1] Ross AG, Bartley PB, Sleigh AC, Olds GR, Li. Y, Williams GM, et al. Schistosomiasis. N Engl J Med 2002;346:121220.

[2] World Health Organization. 1998. Report of the WHO informal consultation on schistosomiasis control [Online].www.who.int/wormcontrol/documents/publications/en/ 992enpdf.

[3] Grobusch MP, Muhlberger N, Jelinek T, Bisoffi Z, Corachan $M$, Harms $G$, et al. Imported schistosomiasis in Europe: Sentinel surveillance data from TropNetEurop. J Travel Med 2003;10:164-9.

[4] Doenhoff MJ, Chiodini PL, Hamilton JV. Specific and sensitive diagnosis of schistosome infection: can it be done with antibodies? Trends Parasitol 2004;20:35-9.

[5] Bierman WF, Wetsteyn JC, van Gool T. Presentation and diagnosis of imported schistosomiasis: relevance of eosinophilia, microscopy for ova and serology. J Travel Med 2005; 12:9-13.

[6] Harries AD, Friatt R, Walker J, Chiodini PL, Bryceson AD. Schistosomiasis in expatriates returning to Britain from the tropics: a controlled study. Lancet 1986;1:86-8.
[7] Whitty CJ, Carroll B, Armstrong M, Dow C, Snashall D, Marshall T, Chiodini PL. Utility of history, examination and laboratory test in screening those returning to Europe from the tropics for parasitic infection. Trop Med Int Health 2000;5:818-23.

[8] Corachan M. Schistosomiasis and international travel. Clin Infect Dis 2002;35:446-50.

[9] Hamilton JV, Klinkert M, Doenhoff MJ. Diagnosis of schistosomiasis: antibody detection, with notes on parasitological and antigen detection methods. Parasitology 1998; 117 (Suppl):41-5.

[10] Al-Sherbiny MM, Osman AM, Hancock K, Deelder AM, Tsang VC. Application of immunodiagnostic assays: detection of antibodies and circulating antigens in human schistosomiasis and correlation with clinical findings. Am J Trop Med Hyg 1999;60:960 -6.

[11] Maddison SE, Slemenda SB, Tsang VC, Pollard RA. Serodiagnosis of Schistosoma mansoni with microsomal adult worm antigen in an enzyme-linked immunosorbent assay using a standard curve developed with a reference serum pool. Am J Trop Med Hyg 1985;34:484-94.

[12] Pardo J, Carranza C, Turrientes MC, Perez Arellano JL, Lopez Velez R, Ramajo V, et al. Utility of Schistosoma bovis adult worm antigens for diagnosis of human schistosomiasis by enzyme-linked immunosorbent assay and electroimmunotransfer blot techniques. Clin Diagn Lab Immunol 2004; 11:1165-70.

[13] Katz N, Chaves A, Pellegrino J. A simple device for quantitative stool tick smear technique in Schistosoma mansoni. Rev Inst Med Trop Sao Paolo 1972;14:393-407.

[14] Peters PAS, Warren KS, Mahmoud AAF. Rapid accurate quantification of schistosome eggs via nucleorope filters. J Parasitol 1976;62:154-5.

[15] López Abán JL, Ramajo V, Pérez Arellano JL, Oleaga A, Hillyer GV, Muro A. A fatty acid binding protein from Fasciola hepatica induced protection in C57/BL mice from challenge infection with Schistosoma bovis. Vet Parasitol 1999;83:107-21.

[16] van Gool T, Vetter H, Vervoort T, Doenhoff MJ, Wetsteyn J, Overbosch D. Serodiagnosis of imported schistosomiasis by a combination of a commercial indirect haemagglutination test with Schistosoma mansoni adult worm antigens and an enzyme-linked immunosorbent assay with S. mansoni egg antigens. J Clin Microbiol 2002;40:3432-7.

[17] Doherty JF, Moody AH, Wright SG. Katayama fever: an acute manifestation of schistosomiasis. Br Med J 1996;313: 1071-2. 
Copyright of Scandinavian Journal of Infectious Diseases is the property of Taylor \& Francis Ltd and its content may not be copied or emailed to multiple sites or posted to a listserv without the copyright holder's express written permission. However, users may print, download, or email articles for individual use. 